\title{
Systemic Targeted Alpha Radiotherapy for Cancer - A Review
}

\author{
Barry J Allen \\ Cancer Pathology \& Cell Biology Laboratory, School of Medicine, \\ University of Western Sydney \\ email: bja1940@optusnet.com.au
}

\begin{abstract}
The fundamental principles of internal targeted alpha therapy for cancer were established many decades ago. The high linear energy transfer (LET) of alpha radiation to the targeted cancer cells causes double strand breaks in DNA. At the same time, the short range of alpha- radiation spares adjacent normal tissues. This targeted approach complements conventional external beam radiotherapy and chemotherapy. Such therapies fail on several fronts, such as lack of control of some primary cancers (eg glioblastoma multiforme) and inhibition of the development of lethal metastatic cancer after successful treatment of the primary cancer.
\end{abstract}

This review charts the developing role of systemic high LET in internal radiation therapy. Targeted alpha therapy is a rapidly advancing experimental therapy that holds promise to deliver high cytotoxicity to targeted cancer cells. Initially thought to be indicated for leukaemia and micrometastases, there is now evidence that solid tumours can also be regressed.

Alpha therapy may be molecular or physiological in its targeting. Alpha emitting radioisotopes such as Bi-212, Bi-213, At-211 and Ac-225 are used to label monoclonal antibodies or proteins that target specific cancer cells. Alternatively, radium-233 is used for palliative therapy of breast and prostate cancers because of its bone seeking properties.

In this review, preclinical studies and clinical trials of alpha therapy are discussed for leukaemia, lymphoma, melanoma, glioblastoma multiforme, bone metastases, ovarian cancer, pancreatic cancer and other cancers.

Keywords: Cancer, Targeted alpha therapy, radiotherapy, cytotoxicity,

\section{Introduction}

This review covers the development of internal, high LET radiotherapy from an Australian perspective; more complete bibliographies are available elsewhere (Elgqvist 2011). Targeted alpha therapy for cancer has progressed from early in vitro studies, through in vivo experiments to phase 1 and 2 clinical trials.

Our initial studies were related to the production and testing of the alpha emitting radioisotope, $\mathrm{Tb}-149$. Other research groups used the accelerator produced At-211. However, the ${ }^{225} \mathrm{Ac}:{ }^{213} \mathrm{Bi}$ generator has become the workhorse for the ongoing research.

Targeted Alpha Therapy (TAT) incorporates the essential elements of immunotherapy of cancer; a targeting molecule that fixes to membrane bound molecules on the surface of cancer cells and a radioisotope label that emits toxic alpha radiation that deposits a large fraction of energy into the targeted cell. There has been a steady rate of in vitro and in vivo alpha publications over the last 25 years, which clearly demonstrated the potential superiority of this therapeutic approach. One paper that stands out was the in vivo mouse study (Bloomer 
1984) for mice with peritoneal ascites, which showed that while alpha radiation could lead to regression of the ascites, beta radiation could not.

This and other papers were the foundations for our extensive alpha research program, which first began with $\mathrm{Tb}-149$, the only lanthanide with a significant alpha decay branching ratio (Allen 1996). At the same time, Memorial Sloan Kettering Cancer Center was already well down the track with the Ac:Bi generator, which has transformed the practicality of TAT. Tb152 was later produced at the ISOLDE facility at CERN and Tb-149 at the Tandem accelerator at ANU (Allen 2000) and later in clinical quantities at the $1 \mathrm{GeV}$ CERN accelerator (Beyer 2002). However, Tb-149 failed the practicality test for clinical applications; ie it could not be readily available at the clinical level.

The use of gamma emitting radioisotopes for imaging is well established in Nuclear Medicine. Radioisotopes such as I-131, I-123, Ga-69, Tl-205 and especially Tc-99m are used to label targeting vectors to allow the pharmacokinetics of radio-conjugates to be determined in human patients via single photon emission computer tomography (SPECT). Positron emission tomography (PET) is developing rapidly as an important diagnostic tool, with F-18 labeled FDG being the main workhorse with PET imaging machines. While most Nuclear Medicine procedures relate to imaging, a small proportion uses I-131, Lu-176 and Y-90 for therapy of cancer. However, the therapeutic efficacy of beta emitting radioisotopes has been found to be limited and applications are more successful in the palliative setting. In recent years, alpha emitting radioisotopes have been used in phase 1 and 2 clinical trials for various cancers. Results generally indicate substantial efficacy well below or at the maximum tolerance dose. It is these studies that are reviewed here.

A number of Symposia on alpha emitting radionuclides in therapy have been held, the most recent being at Berlin (TAT 2011). The principles and practices of targeted alpha therapy (TAT) have been reported (Allen 1999 \& 2006) and most recently and completely in special issues of Current Pharmaceuticals (Elgqvist 2011). The detailed development of the Bismuth alpha emitting radioisotopes for therapy has been reviewed by (Hassfjell 2001).

\section{In Vitro and In Vivo Studies}

The Australian program was based on Bi-213, which is eluted from the Ac-225 generator (Finn 1997). The short half life of Bi-213, $46 \mathrm{~min}$, precludes consideration of long biological life times. Stable alpha-conjugates were synthesised in our laboratory by labeling chelated monoclonal antibodies with Bi-213 to form the alpha immuno-conjugates (AIC). These were tested in vitro and in vivo for melanoma (Allen 2001b, Rizvi 2000), leukaemia (Rizvi 2002), colorectal (Rizvi 2001), prostate (Li 2004a,b), ovarian (Song 2006) and pancreatic cancers (Qu 2005a,b).

The short range of alpha particles, and the short half life of useful alpha emitting radioisotopes are the arguments against TAT being at all effective in regressing tumours (Allen 1999a). Consequently, our studies related to the destruction of isolated cancer cells 
and cell clusters and the inhibition of tumour growth. To this end we developed the 2 day model in mice, where treatment followed 2 days post-inoculation of cancer cells. Mice were then followed until tumours reached $\sim 1 \mathrm{~cm}^{3}$. In all cases, complete inhibition of tumour development was achieved with 75-100 $\mu \mathrm{Ci}$ local subcutaneous (sc) injection (ie in the same location as the cell inoculation). Higher activities were required for systemic (tail vein or intraperitoneal) injection of the AIC. However, efficacy decreases for longer growth times and larger tumours, but can be partially offset by multiple dosing.

Intralesional alpha therapy was performed on human melanoma xenografts in nude mice (Allen, 2001b) and showed complete tumour regression over 4-8 weeks. Intralesional TAT of melanoma with $300 \mu \mathrm{Ci}$ gave complete regression of melanoma xenografts in nude mice, but was far less successful in breast and prostate tumours. These results paved the way for the intralesional phase 1 clinical trial (Allen 2006), wherein the mouse host for the human melanoma was simply exchanged for a human host.

Acute activity tolerances are in the region of $24-36 \mathrm{mCi} / \mathrm{kg}$ for systemic intraperitoneal (ip) injections. However, long term toxicity ( $\sim 6$ months in mice) in the form of delayed radiation nephrosis, reduces the MTD to $\sim 9 \mathrm{mCi} / \mathrm{kg}$ in mice and between 3 and $9 \mathrm{mCi} / \mathrm{kg}$ in rabbits.

While kinetics and bio-distributions will depend on the type of vector used, the melanoma trial provided the basic data to determine specific organ doses for comparison with threshold dose levels and the probability of induced secondary cancer. These data are of considerable value in ensuring patient safety in further systemic phase 1 clinical trials.

\section{PAI2 - uPAR alpha therapy}

The PAI2-uPAR targeting system has several important advantages. First, PAI2 is a human protein, rather than a murine antibody, so overcoming problems of immune response. Second, it is a much smaller targeting molecule so can penetrate tissue more efficiently leading to faster targeting, which is important considering the short half life of the alpha conjugate (47 mins). Finally, pre-clinical studies of over-expression show that uPA is highly expressed in around $75 \%$ of pancreatic adenocarcinomas, using immunohistochemical staining, while expression of uPA mRNA in normal pancreas is only 6\% of that for pancreatic adenocarcinoma (Nielson 2005, Xue 2008, Qu 2005). Thus, although there is frequently a high production of uPAR, which predicts poor survival, but when present, this is countered by a high production of its inhibitor PAI2 improved survival results. Therefore the provision of exogenous PAI2 would not be expected to adversely affect survival.

The human recombinant PAI2 protein was successfully tested in breast (Allen 2003), ovarian ( $\mathrm{Qu}$ 2005, Song 2006,8), prostate ( $\mathrm{Li}$ 2002) and pancreatic (Qu 2005a) cancers. These conjugates are highly selective of and cytotoxic to targeted cancer cells. In vitro cytotoxicity of alpha-conjugates is very much greater than beta conjugates, non-specific alpha-conjugates and free alpha isotope. The lethal pathway for alpha therapy is predominantly apoptosis ( $\mathrm{Li}$ 2004a). 
Preclinical results shown in Figure 1 demonstrate complete inhibition of tumour growth at 4 $\mathrm{mCi} / \mathrm{kg}$ dose at 2 days post-inoculation for local s.c. administration and $9 \mathrm{mCi} / \mathrm{kg}$ dose for systemic administration (Qu 2005). All treated groups showed responses varying from almost complete inhibition to delayed tumour growth compared with controls. However, the low MW of PAI2 means that renal filtration will lead to delayed radiation nephrosis (Allen

\section{Time to end point}

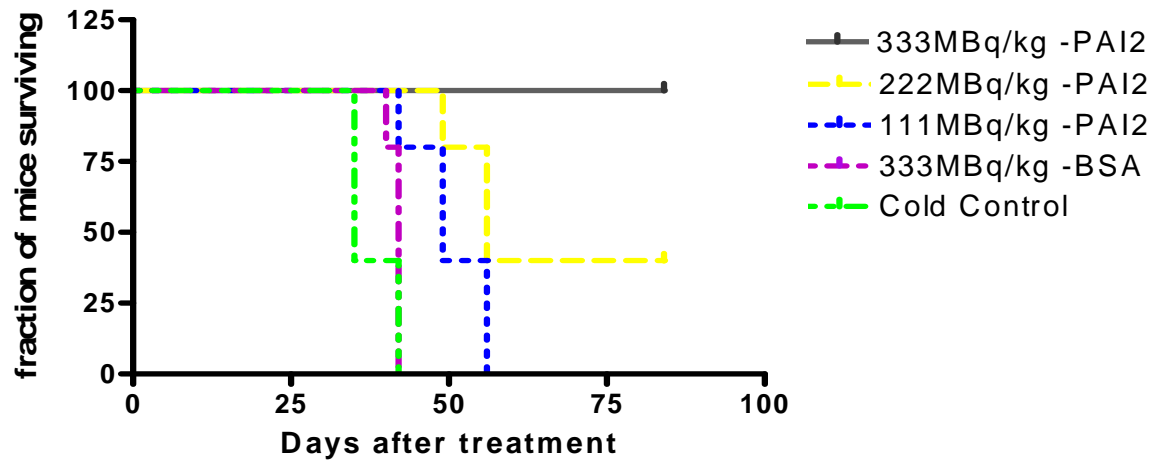

Figure 1: Therapeutic Efficacy: The effect of a single intra-peritoneal injection of ${ }^{213} \mathrm{Bi}-\mathrm{PAI} 2$ on subcutaneous CFPAC-1 human pancreatic cancer xenografts in nude mice. Single i.p injections of 111, 222, $333 \mathrm{MBq} / \mathrm{kg}$ of ${ }^{213} \mathrm{Bi}-\mathrm{PAI} 2 ; 333 \mathrm{MBq} / \mathrm{kg}$ of ${ }^{213} \mathrm{Bi}-\mathrm{BSA}$ (non-targeted hot control) and PAI-2 with no alpha conjugate (cold control) were given two days post-inoculation

2011a).

It is expected that a clinical trial for stage IV pancreatic cancer patients who have either completed or declined standard systemic therapies would soon show efficacy because of the poor prognosis. Any delay in progression of the disease would be of clear benefit to the patient. Systemic targeted alpha therapy has the potential to regress pancreatic cancers and to eliminate micrometastases. It is expected that TAT would be indicated for the control of pancreatic cancer after resection of the primary tumour, when complete cell kill of micrometastases could be achieved, leading to elimination of clinical disease.

\section{C595 anti-mucin alpha therapy}

C595 is an IgG3, murine monoclonal antibody raised against the protein ce human urinary epithelial mucin (MUC1). MUC1 is found to be frequently upregulated and abnormally glycosylated in a number of common malignancies, including breast bladder, colon, ovarian, prostate and gastric cancer. Cancer-associated MUC1 is structu] lifferent to normal MUC1 in that the former has shorter and less dense O-glycan cuanus, which exposes novel regions of the protein core.

The expression of tumor-associated antigen mucin-1 (MUC-1) on breast, prostate, ovarian and pancreatic cancer cell lines, in cell clusters and animal xenografts was detected by indirect immmuno-staining. Monoclonal antibodies (MAbs) C595 (test) and A2 (non-specific 
control) were labeled with ${ }^{213} \mathrm{Bi}$ using the chelator $\mathrm{CHX}-\mathrm{A}$ " to form the alphaimmunoconjugate (AIC).

Preclinical results show inhibition of tumour growth and regression of cell clusters. Over 90\% of primary prostate, pancreatic and ovarian tumours expressed MUC1 while $95 \%$ of normal tissues did not (Qu 2004, Li 2004a, Song 2008b, Allen 2011a). Further, MUC1 expression was found on the surface of cancer cell lines. The lethal pathway in all in vitro studies after TAT was found to be predominantly by apoptosis.

\section{Clinical Trials}

A great deal of preclinical work paved the way for the advance to clinical trials in recent years. The Sloan Kettering Memorial Cancer Center has led the way, first with the application of Bi-213 immunotherapy and later with Ac-225. Other laboratories have concentrated on Bi-212 and At-211. The advantages of the Bi radioisotopes are that they can be generated from long lived parents, Ac-225 with $10 \mathrm{~d}$ and Th-228 with $1.91 \mathrm{y}$ half lives, which can be imported from overseas. The Ac-Bi generator has an additional advantage in that it decays in house and does not need long term waste disposal. At-211, with a $7 \mathrm{~h}$ half life, needs to be used at or near the production site.

While the half lives of Bi-213 (47 minutes) and Bi-212 (61 minutes) are rather short, there is sufficient time for synthesis of the alpha-immuno-conjugates, and for vascular distribution throughout the body. However, there is inadequate time for infusion into tumours, which can take 24-48 hours. This is one reason for the development of the Ac-225 alpha-conjugate, as the $10 \mathrm{~d}$ half life allows plenty of time for infusion through the target tumours. On the other hand, the short range of the alpha products requires a high degree of homogeneity if all tumour cells are to be neutralised.

Targeting vectors must be specific for the cancers to be treated. As such, a number of vectors are being used or are to be introduced into the clinic. The following monoclonal antibodies (MAb) are in use: humanised HuM195 targets acute myelogenous leukaemia; the murine 9.2.27 targets the MCSP antigen on melanoma cells and GBM cells; the anti-CD20 for lymphoma; MX35 F(ab')2 for ovarian cancer; and the human-mouse chimeric anti-tenascin $81 \mathrm{C} 6$ for GBM. In the case of bone cancer, $\mathrm{RaCl}_{2}$ has a natural affinity for bone. Other proposed vectors are PAI2 against uPA, which is widely expressed by many cancers at their most malignant stage, and C595 a murine MAB against MUC-1, also of generic nature. The polysaccharide capsule binding MAb $18 \mathrm{~B} 7$ is proposed for fungal infection.

Seven clinical trials were reported at the Berlin TAT symposium (TAT, 2011). Bi-213 was used for studies in acute myelogenous leukaemia (AML), melanoma and lymphoma; Ac225 for AML; Ra-223 for bone cancer and At-211 for GBM and ovarian cancer. The Phase $1 \mathrm{Bi}-$ 213 trial for AML has been completed and the current trial is phase 2 with chemotherapy pretreatment. The intralesional melanoma trial with $\mathrm{Bi}-213$ has also been completed, being followed by a systemic trial with the same alpha conjugate. The following sections review the results of past and current clinical trials, and the objectives of proposed trials. 
Current and completed clinical trials are as follows:

- Completed Phase I study for acute myeloid leukaemia (AML) (Jurcic, 2002)

- Ongoing phase II study for post-chemotherapy of AML (Jurcic, 2011)

- Ongoing phase I study with Ac-225 (Jurcic, 2011)

- Completed phase 1 trial for intralesional melanoma (Allen, 2006)

- Phase 1 trial of systemic melanoma (Raja,2007; Allen 2008, 2011b),

- Completed phase 1 trial of Glioblastoma (Zalutsky, 2005),

- Competed pilot trial of GBM (Cordier, 2010)

- Completed trial of Ra-223 for bone metastases (Nilsson, 2005),

- Phase 1 for lymphoma (Miederer, 2003).

- Phase 1 trial in GEP-NET (Kratochwil, 2011).

While solid tumours have never been envisaged as suitable targets for TAT, in contrast with liquid cancers and micrometastases (Allen, 1999a), stage 4 advanced cancer patients are used in phase 1 trials for toxicity studies.

The intralesional melanoma trial and our current systemic melanoma trial use the 9.2.29 mab to target the melanoma-associated chondroitin sulfate proteoglycan (MCSP) receptor expressed by lesions of more than $90 \%$ of melanoma patients. This antigen is the same as the HMWMAA and thought to be identical with the NG2 murine anitgen. The antibody is covalently coupled to the cDTPA chelator, and labeled with the Bi-213 alpha emitting radioisotope. The objective of these phase 1 trials with stage 4 melanoma patients was to determine the safety of the AIC, and so far complications of any type or level have not been observed up to $25 \mathrm{mCi}$. However, unexpected tumour regressions have been observed at quite low doses, such that a new concept was introduced to explain the clinical responses observed after systemic alpha therapy, called tumour anti-vascular alpha therapy (TAVAT) (Allen 2007). Leaky neogenic capillaries allow extra-vascular diffusion of the AIC to target antigens on contiguous pericytes and cancer cells. Alpha emission kills the capillary endothelial cells, shutting down the capillaries with subsequent starvation of the tumour.

\section{Acute myelogenous leukaemia (AML)}

The feasibility, safety and anti-leukaemic activity of the AIC, ${ }^{213} \mathrm{Bi}-\mathrm{CHX}-\mathrm{A}$ "-HuM195 was demonstrated in stage 4 subjects with AML (Jurcic 2002). 18 patients with relapsed and refractory acute myelogenous leukaemia or chronic myelmonocytic leukaemia were treated with 10.36 to $37 \mathrm{MBq} / \mathrm{kg}$ of AIC. No significant extramedullary cytotoxicity was observed, but all 17 evaluable subjects developed myelsuppression, with $22 \mathrm{~d}$ median recovery time. The AIC localised rapidly within 10 minutes and was retained in areas of leukaemic involvement, including the bone marrow, liver, and spleen, as shown in Figure 2 below.

Absorbed dose ratios for these sites compared to normal tissue were 1000 times greater than for beta-emitting conjugates. $93 \%$ of subjects experienced reductions in circulating blasts, 
and $78 \%$ had reductions in bone marrow blasts. This first alpha therapy trial in humans showed that the approach was safe, feasible and efficacious.

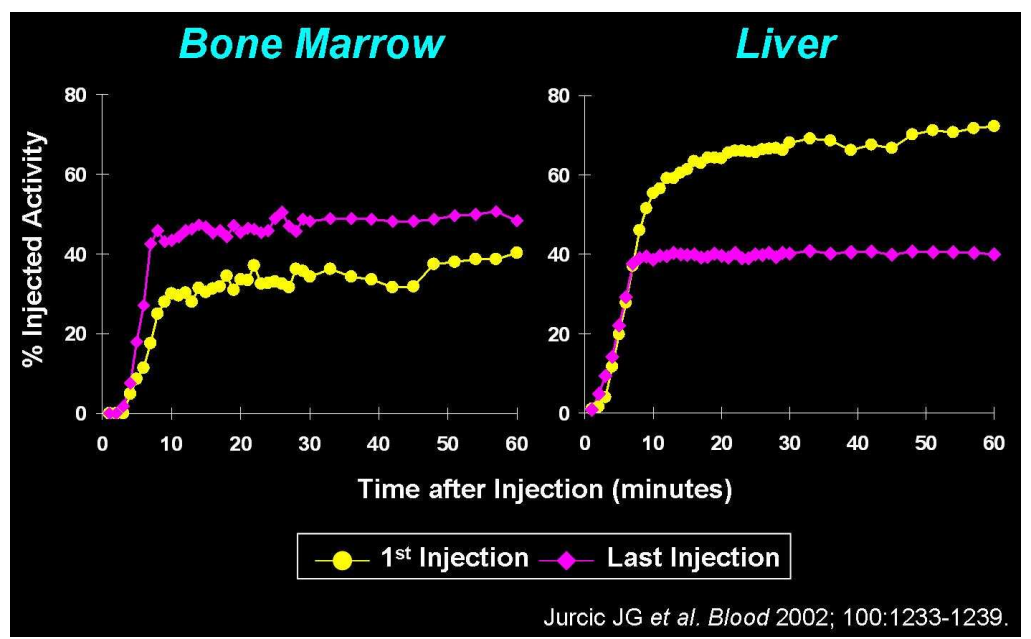

Figure 2: Uptake of the AIC as a function of time in bone marrow and liver. Saturation occurs within 10 minutes post-injection.

\section{AML phase 1\&2}

The phase 1 study reported in section 3.1 showed that while massive cell kill could be achieved with TAT, the tumour load $(\sim 1 \mathrm{~kg})$ was far too high for control to be achieved. A phase 1/2 trial was implemented that uses partial cyto-reduction with cytarabine (200 $\mathrm{mg} / \mathrm{m}^{2} /$ day for 5 days) followed by 0.5 to $1.25 \mathrm{mCi} / \mathrm{kg}$ of AIC. The MTD was $1 \mathrm{mCi} / \mathrm{kg}$, the dose limiting toxicity being myelosupression. 6 of 25 subjects $(24 \%)$ responded at $>=1.0$ $\mathrm{mCi} / \mathrm{kg}$, with $2 \mathrm{CRs}$ lasting 9 and 12 months, 2 CRp lasting 2 and 5 months, and 2 PRs lasting 4 and 7 months (TAT 2011).

\section{Intralesional metastatic melanoma}

The aim was to develop and implement intralesional targeted alpha therapy (ITAT) for metastatic melanoma, being the first part of a program to establish a new systemic therapy. The benign targeting vector 9.2.27 was labeled with ${ }^{213} \mathrm{Bi}$ to form the alphaimmunoconjugate ${ }^{213} \mathrm{Bi}$-cDTPA-9.2.27 (AIC), which is highly cytotoxic to targeted melanoma cells (Allen 2001).

The safety and efficacy of intralesional AIC in patients with metastatic skin melanoma was investigated in 16 melanoma patients, all with melanomas that were positive to the monoclonal antibody 9.2.27 (Allen 2005). AIC doses from 50 to $450 \mu \mathrm{Ci}$ were injected into lesions of different sizes, causing massive tumour cell death as observed by the presence of tumour debris. The AIC was very effective in delivering a high dose to the tumour while sparing other tissues. There were no significant changes in blood proteins and electrolytes. There was no evidence of a human-antimouse-antibody reaction. Evidence of significant 
decline in serum marker melanoma-inhibitory-activity protein (MIA) at 2 weeks post-TAT was observed.

Intralesional TAT for melanoma in human patients was found to be safe and efficacious to $1350 \mu \mathrm{Ci}$ (Allen, 2006). Tumours were resected at 8 weeks post-ITAT, to show massive cell debris in the injected volume, but no effect in untreated tumour or in the antibody only treated tumour in the same patient. Tumour to kidney activity ratios were $~ 3000$. MIA, apoptosis and ki67 proliferation marker tests all indicated that TAT is a promising therapy for the control of inoperable secondary melanoma or primary ocular melanoma. As such, intralesional TAT is indicated for uveal melanoma and brain metastases.

\section{Bone metastases from breast and prostate cancers- phase 1 trial}

${ }^{223} \mathrm{Ra}$ is a bone seeking alpha emitter with potential for palliating breast and prostate cancer metastatic to the bone. A phase 1 trial has been reported for 15 hormone refractory prostate cancer patients and 10 breast cancer patients, all with metastatic bone disease (Nilsson 2005). Activities of 50 to $250 \mathrm{kBq} / \mathrm{kg}$ were well tolerated; $2 / 25$ subjects experienced grade 3 leucopenia; there was no grade $2+$ thrombocytopenia and no dose limiting toxicity. 10/25 subjects suffered diarrhoea. Evidence of efficacy was found with substantial reductions in serum alkaline phosphatase (ALP) and improved pain control, but was not dose dependent.

\section{Bone metastases from prostate cancers- phase II trial}

A randomised, double blind, placebo controlled, multicentre phase II study investigated the effect of multiple doses of Ra-223 in subject with symptomatic hormone-refractory prostate cancer (Nilsson 2007). Efficacy endpoints were the reduction in bone-specific ALP concentration and time to occurrence of skeletal-related events (SRE).

Patients due to receive external beam radiotherapy for pain relief were randomly assigned to 4 monthly Ra-223 injections or saline injections, in parallel with external beam radiotherapy. Subjects were monitored for survival and long term toxicity out to 24 months. Confirmed PSA response was defined as a $50 \%$ reduction from baseline; PSA progression a $25 \%$ increase from the nadir and 50\% increase for those with a confirmed PSA response. 64 patients were recruited into the trial, 33 being assigned to XBRT plus Ra-223. Baseline values for both groups were not significantly different, nor were adverse events. However, the Ra-223 group had significant reductions in all five markers, ie bone-ALP, total-ALP, PINP, CTX-1 and ICTP. Significant differences were observed with changes in PSA from baseline to 4 weeks, PSA decreasing by $24 \%$ in the Ra- 223 group and increasing by $45 \%$ in the placebo group. Median time to progression of PSA and survival was 26 weeks and 65 weeks for Ra-223, compared with 8 weeks and 46 weeks for placebo ( $\mathrm{P}=0.04$ and 0.07$)$.

Ra-223 was fast tracked by the FDA and is now the first alpha therapy to be approved for clinical application, specifically for palliation of bone metastases from prostate cancer. 


\section{Glioblastoma Multiforme (GBM)}

The first clinical application of At-211 in humans involved the injection into the resection cavity of escalating doses of ${ }^{211}$ At-human antimouse chimeric anti-tenascin MAb 81C6. (Zalutsky, 2005). Injected activities ranged from 2 to $10 \mathrm{mCi}$, but the MTD was not reached. However, 6 of 17 subjects experienced grade 2 neurotoxicity at 6 weeks, which fully resolved in all but one case. Radionecrosis was not observed. The median delivered dose was 2800 Gy, giving a median survival for GBM subjects $(\mathrm{N}=14)$ of 52 weeks and 116 weeks for anaplastic oligodendroglioma $(\mathrm{N}=3)$. These results compare favourably with 36 weeks median survival after diagnosis for standard therapy. Regional administration of ${ }^{211}$ Atch81C6 was found to be feasible, safe and efficacious.

\section{Glioma}

Critically located gliomas represent a challenging subgroup of intrinsic brain neoplasms because radical treatment and preservation of neurological function are contrary goals. The successful targeting of gliomas with locally injected ${ }^{90}$ Y-DOTAGA-substance P was not indicated for critically located tumours, where the mean beta range of $5 \mathrm{~mm}$ may seriously damage adjacent brain areas. ${ }^{213} \mathrm{Bi}$ emits alpha radiation with a mean range of 81 $\mu \mathrm{m}$ and may have a more favourable toxicity profile. Five patients with critically located gliomas (WHO grades II-IV) were locally injected with ${ }^{213} \mathrm{Bi}$-DOTA-substance P in a pilot study (Cordier 2010).

Targeted radio-peptide therapy using ${ }^{213} \mathrm{Bi}$-DOTA-substance $\mathrm{P}$ was found to be feasible and tolerated without additional neurological deficit. No local or systemic toxicity was observed. ${ }^{213} \mathrm{Bi}$-DOTA-substance $\mathrm{P}$ showed high retention at the target site. MR imaging was suggestive of radiation induced necrosis and demarcation of the tumours, which was validated by subsequent resection. This study provided proof of concept that targeted local radiotherapy using ${ }^{213} \mathrm{Bi}$-DOTA-substance $\mathrm{P}$ is feasible and may represent an innovative and effective treatment for critically located gliomas. Primarily non-operable gliomas may become resectable with this treatment, possibly improving prognosis.

\section{Lymphoma}

12 subjects with relapsed or refractory non-Hodgkins lymphoma (NHL) have been treated so far with $28,33,39$ and $44 \mathrm{MBq} / \mathrm{kg}$ of ${ }^{213} \mathrm{Bi}$-anti-CD20, without evidence of short term toxicity. Delayed toxicity was experienced by 5 subjects with myelosuppression and one subject with fever. The dose limiting organ is bone marrow, which received 3.3 to 7.2 $\mathrm{mGy} / \mathrm{MBq}$ (Schmidt, 2004).

\section{Ovarian Cancer}

The $\alpha$-emitter ${ }^{211}$ At labeled to a monoclonal antibody has proven safe and effective in treating microscopic ovarian cancer in the abdominal cavity of mice (Andersson 2009). Women in complete clinical remission after second-line chemotherapy for recurrent ovarian carcinoma were enrolled in a phase I study. The aim was to determine the pharmacokinetics for assessing absorbed dose to normal tissues and investigating toxicity. 
Nine patients underwent laparoscopy $2-5 \mathrm{~d}$ before the therapy; a peritoneal catheter was inserted, and the abdominal cavity was inspected to exclude the presence of macroscopic tumor growth or major adhesions. ${ }^{211}$ At was labeled to $\mathrm{MX} 35 \mathrm{~F}\left(\mathrm{ab}^{\prime}\right)_{2}$ using the reagent $N$ succinimidyl-3-(trimethylstannyl)-benzoate. Patients were infused with ${ }^{211} \mathrm{At}-\mathrm{MX} 35 \mathrm{~F}\left(\mathrm{ab}^{\prime}\right)_{2}$ (22.4-101 MBq/L) in dialysis solution via the peritoneal catheter. Samples of blood, urine, and peritoneal fluid were collected at 1-48 h. Hematology, renal and thyroid function were followed for a median of 23 months.

In terms of the initial activity concentration (IC) of the infused solution, the decay-corrected activity concentration decreased with time in the peritoneal fluid to $50 \%$ IC at $24 \mathrm{~h}$, increased in serum to $6 \%$ IC at $45 \mathrm{~h}$, and increased in the thyroid to $127 \% \pm 63 \%$ IC at $20 \mathrm{~h}$ without blocking and less than $20 \%$ IC with blocking. No other organ uptakes could be detected. The estimated absorbed dose to the peritoneum was $15.6 \pm 1.0 \mathrm{mGy} /(\mathrm{MBq} / \mathrm{L})$, to red bone marrow it was $0.14 \pm 0.04 \mathrm{mGy} /(\mathrm{MBq} / \mathrm{L})$, to the urinary bladder wall it was $0.77 \pm 0.19$ $\mathrm{mGy} /(\mathrm{MBq} / \mathrm{L})$, to the unblocked thyroid it was $24.7 \pm 11.1 \mathrm{mGy} /(\mathrm{MBq} / \mathrm{L})$, and to the blocked thyroid it was $1.4 \pm 1.6 \mathrm{mGy} /(\mathrm{MBq} / \mathrm{L})$ (mean $\pm \mathrm{SD})$. No adverse effects were observed. Intraperitoneal administration of ${ }^{211} \mathrm{At}-\mathrm{MX} 35 \mathrm{~F}\left(\mathrm{ab}^{\prime}\right)_{2}$ could achieve therapeutic absorbed doses in microscopic tumor clusters without significant toxicity.

\section{$4 \quad$ Systemic therapy for metastatic melanoma}

The aim of this Australian study was to assess toxicity and response of systemic alpha therapy for metastatic melanoma using the alpha-immunoconjugate ${ }^{213}$ Bi-cDTPA-9.2.27 (Raja, 2007). Tools used to investigate the responses were, physical examination, imaging of tumours, pathology comparisons over 12 weeks, GFR, CT comparisons and changes in tumour marker over 8 weeks. Responses are based on RECIST criteria.

40 patients with stage IV melanoma/ in-transit metastasis were treated with activities of 55-947 MBq. Using RECIST criteria $50 \%$ of subjects experienced stable disease and $12 \%$ showed partial response. One patient showed near complete response after a $5 \mathrm{mCi}$ IV injection of the AIC (20/21 lesions completely disappeared, see Figure 3) and was retreated at 12 months because of an excellent response to the initial treatment. Another patient showed response in his tumour on mandible and reduction in lung lesions. $30 \%$ of patients experienced progressive disease over 8 weeks, and all subjects eventually progressed and succumbed to the disease.

The tumour marker melanoma inhibitory activity protein (MIA) reduced over 8 weeks in most patients. However, there was a disparity of dose with responders. Toxicity at any level was not observed over the range of administered activities. 
The observation of responses without any toxicity indicates that targeted alpha therapy has the potential to be a safe and effective therapeutic approach for metastatic melanoma.

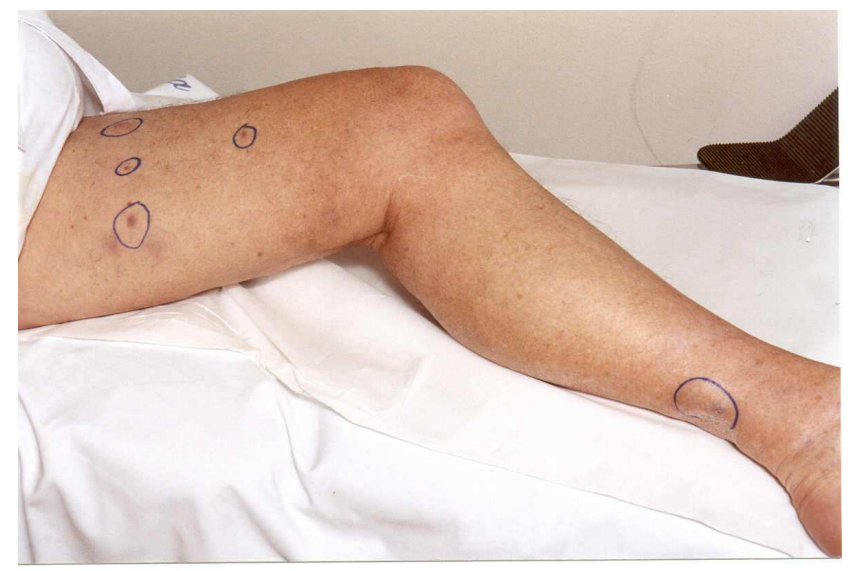

Figure 3: 20 of 21 melanomas completely regressed after systemic TAT. The initial sizes of the larger tumours are shown as blue rings. Post-TAT tumour beds revealed a complete absence of viable melanoma cells.

The observation of efficacy at quite low doses showed that this trial, while adequate as phase 1 , was inadequate to investigate the underlying factors that were contributing to the unexpected efficacy. As such, the trial was terminated in June 2007 without reaching the MTD and a new trial was designed to provide more detailed information.

\section{Improvements to the trial}

The earlier trial used the cDTPA chelator to link the antibody and radioisotope, as this was the only commercially available chelator at that time. As delayed radiation nephrosis is the main concern, CHX-A", being more stable, is expected to reduce the renal uptake of free Bi213 and so increase the maximum tolerance dose for the kidneys. Further, commercial production of CHX-A" is now available (Macrocyclics USA).

The monoclonal antibody 9.2.27 targets the MCSP antigen and if expression is low, antigens can be more readily saturated and blocked by unlabelled antibody, thus limiting tumour regression. One way around this problem is to increase the specific activity (SA) of the AIC. The AIC is usually prepared by minimizing the free radio-isotope in the labeling process. However, our objective is to minimize the unlabelled antibody fraction. This then leads to a higher SA, less blocking of target antigens, and more effective therapy. Further, the higher specific activity will reduce the amount of antibody injected, even at higher activities, therefore reducing the HAMA effect.

Dose limiting toxicity will be defined in terms of renal function; GFR Grade1 (normalized for age) or Grade 2 serum creatinine. GFR will be measured at $0,26,52$ and 78 weeks. If there is $>25 \%$ decline GFR will be repeated in one month for verification. Serum creatinine will be measured at each visit.

A single administration of AIC was given in the first trial, (if needed 2-3 injections on the same day to achieve the required injected activity). With the higher activities, a fractionated 
dose regime will be more practical, and may give improved efficacy as tumours capillaries may be damaged, resulting in increased permeability for the AIC. Daily fractionation over 45 days would not be of concern for immune response (HAMA) as the time period is too short for the generation of an immune response (7-14 Days).

Blocks of previous biopsies will be obtained with the consent of the patient to assess the expression of the targeted antigen MCSP. Tumour biopsy may be taken to observe the effect of the therapy.

Biological dosimetry will be obtained by observation of radiation damage to peripheral blood lymphocytes (Song 2007a). Radiation causes the formation of micronuclei in lymphocytes, which can be counted before and after treatment with blood samples.

\section{Discussion}

Alpha therapy for AML is very effective in reducing the AML cancer cell load. When used with prior chemotherapy at the MTD level, some important complete and partial responses are observed.

Patient data for NHL are not as encouraging, and it is not yet clear if responses will be observed at the MTD.

In the case of intralesional TAT, quite low injected activities $(<0.5 \mathrm{mCi})$ can bring about tumour regression. Even with systemic therapy, and contrary to expectations, melanomas have been completely regressed without recurrence with systemic administration of $<10 \mathrm{mCi}$ of AIC. There is no evidence of any adverse events up to $25 \mathrm{mCi}$. The ability to regress solid tumours was unexpected, and is explained by tumour anti-vascular alpha therapy (TAVAT), as hypothesised by Allen et al (2007). The diffusion of AIC by leaky tumour capillaries into the peri-capillary space allows the antigens of pericytes and contiguous cancer cells to be targeted, from which alpha rays can kill endothelial cells, leading to closure of the capillaries. If enough capillaries are closed down, then the tumour may regress. The variable tumour capillary permeability is expected to be the major determining factor in TAVAT.

Monte Carlo calculations of the microdosimetry (Huang, 2011) show that TAVAT is theoretically possible in terms of Bi-213 concentrations and the probability of alpha crossings of the endothelial cell nucleus.

Intra-cavity administration of the AIC for GBM shows improved survival of 52 weeks without serious adverse events. This approach holds great promise for improving prognosis of this fatal disease.

Palliative therapy with Ra-223 appears promising for breast and prostate cancer metastatic to the bone. When used as therapy adjunctive to external beam radiotherapy, marked reductions in PSA are seen for prostate cancer. 
Intraperitoneal administration of the AIC for ovarian cancer may also be effective, and so far has not induced any adverse events.

A number of preclinical studies should lead to new clinical trials. Among these is the use of PAI2 as a targeting vector for pancreatic cancer. Being a small molecule (MW=47 kD), alpha-PAI2 may more easily diffuse through tumour capillary fenestrations to target cancer cells, and set up a TAVAT effect. On the down side is the higher renal uptake arising from the lower MW. PAI2 targets UPA, a generic receptor expressed by many cancers. Also generic in nature is the MUC-1 receptor, targeted by the MAb c595.

TAT could have application for the control of microbial disease and for AIDS. The potential role of alpha therapy has been explored for fungal disease, the human pathogens Cryptococcus neoformans and Histoplasma capsulatum, and pneumococcal infection and viral disease (Dadachova 2006).

Of some concern is the impact of second cancers, arising from point mutations from stochastic radiation damage to chromosomes and incorrect radiation damage repair. The high radiation weighting factor for alpha particles $\left(\mathrm{R}_{\mathrm{w}}=20\right)$ could limit the application of TAT to end stage cancers. The mutagenic potential of ${ }^{213} \mathrm{Bi}$ conjugated to a human melanoma antigen-specific antibody (9.2.27) was examined using an in vivo transgenic mouse model containing multiple copies of a lacZ target gene in every cell, allowing the quantification and comparison of mutagenesis in different organs (Allen, 2009). The mutant frequency and mutant spectra were analysed for the brain, spleen and kidneys. The brain and spleen did not show significant increases in induced mutation frequencies compared to spontaneous background levels or changes in mutant spectra, these results being independent of p53 status. However, elevated mutation frequencies and persistent size change mutations were observed in the kidneys, but were not significant $(\mathrm{P}=0.05)$. The effect of p53 status was also evident, as p53 heterozygotes displayed higher mutation frequencies than their wild-type counterparts, suggesting a reduction in the p53 gene may lead to an increased susceptibility to mutagenesis. These effects were time dependent and levels returned to those of the controls at 4 weeks post-irradiation, albeit with a predominant residue of size mutations. However, these mutations were observed at activities very much higher than those expected for the therapy of human patients. As such, the induction of secondary cancer with the ${ }^{213} \mathrm{Bi}$-cDTPA9.2.27 alpha immunoconjugate is not expected to be a significant problem in the clinic.

The objectives in the application of targeted alpha therapy (TAT) for cancer therapy relate to the elimination of isolated cancer cells, cell clusters and tumours. Requirements for isolated cancer cells are good cellular targeting, high specific activity and very short range to spare normal tissue. The regression of cell clusters in the peri-vascular space requires high capillary permeability and short range cross fire whereas for developed tumours, good bioavailability and anti-capillary activity are essential (Allen 2011c). 
Of the current sources of alpha radiation, the Ac:Bi generator is the most practical, bringing therapy to Nuclear Medicine with the same practicality as the Mo:Tc generator has for imaging.

\section{Conclusions}

Alpha therapy is still a work in progress, but great gains are being made in translating from preclinical studies to clinical trials. Ideally suited to leukaemia, alpha therapy is demonstrating efficacy, but at the MTD. GBM results from intra-cavity administration are very promising, with 52 week median survival. However, the promise of targeted alpha therapy is greatly extended by the development of TAVAT for solid tumours. Metastatic melanoma results show surprising tumour regressions at doses very much below the MTD, and if further research is successful, could change the prognosis for end-stage cancers. More studies are needed in the fields of dose normalization, real time microdosimetry and biological dosimetry for deterministic and stochastic effects.

\section{Acknowledgements}

The TAT project needs the skills of many colleagues; Drs S Rizvi in radiochemistry, C Raja in clinical trials, Y Li in immunology and past and present PhD students S Rizvi, CF Qu, EY Song and CY Huang. Clinical collaborators were Professors P Graham, J Kearsley at St George Hospital, R Smith at Royal North Shore Hospital and J Thompson at Royal Prince Alfred Hospital. Our project depends heavily on support from Drs A Morgenstern and C Apostolidis at the Institute for Transuranium Elements (ITU), Germany.

These acknowledgements would not be complete without noting the extensive grant support for our TAT program by the USDOD, which has complemented the lack of support from Australian funding agencies.

\section{References}

Allen BJ and Blagojevic N, 1996, Alpha and beta emitting radiolanthanides in targeted cancer therapy: the potential role of terbium-149 Nucl Med Communications 17, 40-47.

Allen BJ. 1999a. Can alpha-immunotherapy succeed where other systemic modalities have failed? Nucl Med Communications, 20, 205-207.

Allen BJ 1999b. Targeted alpha therapy: evidence for efficacy of alpha immunoconjugates in the management of micrometastatic cancer. Australas Radiology., 43, 480-486.

Allen BJ, Goozee G., Sarkar S, Beyer G, Morel, C \& Byrne. 2000. A.P. Production of terbium-152 by heavy ion reactions and proton induced spallation. Applied Radiat Isotopes, 54, 53-8.

Allen BJ, Rizvi, S.M. \& Tian, Z. 2001. Preclinical targeted alpha therapy for subcutaneous melanoma. Melanoma Res, 11, 175-82.

Allen B.J., Tian, Z., Rizvi, S.M.A., Li, Y. \& Ranson, M. 2003. Preclinical studies of targeted alpha therapy for breast cancer using ${ }^{213} \mathrm{Bi}$-labelled-plasminogen activator inhibitor type 2. Brit J Cancer, 88, $944-50$. 
Allen BJ, Raja, C., Rizvi, S., Li, Y., Tsui, W., Zhang, D., Song, E., Qu, C.F., Kearsley, J., Graham, P. \& Thompson, J. 2004. Targeted alpha therapy for cancer. Phys Med Biol, 49, 3703-12.

Allen BJ, Raja, C, Rizvi, SMA, Li Y, Tsui W, Graham P, Thompson JF, Reisfeld RA, Kearsley JH, Morgenstern A, Apostolidis C 2005. Intralesional targeted alpha therapy for metastatic melanoma. Cancer Biol Therapy, 4, 1318-24.

Allen, BJ. 2006 Internal High LET Targeted Radiotherapy for Cancer. Phys. Med. Biol. 5. R327-R341

Allen BJ, Raja C, Rizvi, SMA et al. 2006. Intralesional targeted alpha therapy for metastatic melanoma. . Cancer Biol Therapy, 4, 1318-24.

Allen BJ, Raja C, Rizvi SMA, Song EY, Graham P. 2007. Tumour anti-vascular alpha therapy: a mechanism for the regression of solid tumours in metastatic cancer. Phys. Med. Biol. 52 L15-L19

Allen BJ, Raja C, Rizvi SMA, Graham P, Kearsley JH. 2008. New Directions for Clinical Trials of Targeted Alpha Therapy for Metastatic Melanoma. Current Radiopharmaceuticals 1, 240-250.

Allen BJ, So T, Rizvi SMA, Song EY, Fernandez HR, Lutz-Mann L. 2009. Mutagenesis induced by targeted alpha therapy using ${ }^{213} \mathrm{Bi}-\mathrm{cDTPa}-9.2 .27$ in lac $Z$ transgenic mice. Cancer Biol Therapy 8, 9, 777-781.

Allen BJ, Rizvi SMA, Qu CF, Smith R. 2011a. Targeted alpha therapy approach to the management of pancreatic cancer. Cancers: Special issue "Pancreatic Cancer" 3, 1821-1843.

Allen BJ, Singla AA, Abbas Rizvi SM, Graham P, Bruchertseifer F, Apostolidis C, Morgenstern A. 2011b. Analysis of Patient Survival in a Phase 1 Trial of Systemic Targeted Alpha Therapy for Metastatic Melanoma. Immmunotherapy 3(9), 1041-1050.

Allen BJ. 2011C. Future Prospects for Targeted Alpha Therapy. Current Radiopharmaceuticals 4(4):336-342.

Andersson H, Cederkrantz E, Bäck T, Divgi C, Elgqvist J, Himmelman J, Horvath G, Jacobsson L, Jensen H, Lindegren S, Palm S, Hultborn R. 2009. Intraperitoneal alpha-particle radioimmunotherapy of ovarian cancer patients: pharmacokinetics and dosimetry of ${ }^{211}$ At-MX35 F(ab')2--a phase I study. $J$ Nuclear Medicine 50 (7) s. 1153-60.

Beyer, G.J., Comor,J J, Dakovic, M, Soloviev,D, Tamburella,C, Hagebo,E, Allen,B J, Dmitriev,S N, Zaitseva, N G, ISOLDE Collaboration. (2002) Production routes of the alpha-emitting ${ }^{149} \mathrm{~Tb}$ for medical application. Radiochim Acta, 90, 247-52.

Bloomer, W.D., McLaughlin,W H,Atcher,R W, et al. (1984). ${ }^{211}$ At radiocolloid therapy: further observations and comparison with radiocoloids of ${ }^{32} \mathrm{P},{ }^{165} \mathrm{Dy}$, and ${ }^{90} \mathrm{Y}$. Int J Radiat Oncol Biol Phys, 10, 341-8.

Cordier D, Forrer F, Bruchertseifer F, Morgenstern A, Apostolidis C, Good S, Mullerp-rand J, Maecki H, Reubi JC, Merlo A (2010) Targeted alpha-radionuclide therapy of functionally critically located gliomas with ${ }^{213} \mathrm{Bi}-\mathrm{DOTA}$-[Thi8,Met(O2)11]-substance P: a pilot trial. Eur J Nucl Med Mol Imagimg $37,1335-1344$

Cozzi PJ, Wang J, Delprado W, Perkins AC, Allen BJ, Russell PJ, Li Y. (2005) MUC1, MUC2, MUC4, MUC5AC and MUC6 expression in the progression of prostate cancer. Clin Exp Metastases $22 ; 565-573$ 
Dadachova E, Patel MC, Toussi S et al (2006) Targeted killing of virally infected cells by radiolabelled antibodies to viral proteins. PLoS Medicine 2 (11) e427

Elgqvist J, Editor, (2011) Current Radiopharmaceuticals 4 (issues 3 and 4)

Finn,RD, McDevitt, MR, Scheinberg,DA et al. (1997). Refinements and improvements for Bi-213 production and use as a targeted therapeutic radiopharmaceutical. J Labelled Compds Radiopharm $\mathbf{4 0 ,}$ 293.

Hassfjell, S., Brechbiel,M W. (2001). The development of the alpha-particle emitting radionuclides ${ }^{212} \mathrm{Bi}$ and ${ }^{213} \mathrm{Bi}$, and their decay chain related radionuclides, for therapeutic applications. Chem Rev 101, 2019-36.

Huang CY, Guatelli S, Oborn BM, Allen BJ (2010) Background dose for systemic targeted alpha therapy. Joint Int. Conf. Supercomputing Nucl .Applic. and Monte Carlo, Tokyo Oct 17-21.

Jurcic J, Rosenblat TL, McDevitt MR, Pandit-Taskar N, Carrasquillo J, Divgi CR, Sqouros G, Morgenstern A, Cicic D, Larson SM, Scheinberg DA. (2011) Alpha-particle immunotherapy for acute myeloid leukaemia with Bismuth-213 and actinium-225. 7th Symposium on targeted Alpha Therapy, Berlin July 17-19.

Kratochwil C, Giesel FL, Bruchertseifer F, Apostolidis C, Mier W, Morgenstern A, Haberkorn U. (2011) Dose escalation study of peptide receptor alpha-therapy with arterially administered ${ }^{213} \mathrm{Bi}$ DOTATOC in GEP-NET patients refractory to beta-emitters. Ann. Congress European Assoc. Nuclear Medicine, Oct 15-19 Birmingham.

Li, Y., Rizvi, S.M.A., Ranson, M. \& Allen BJ (2002). 213Bi-PAI2 conjugate selectively induces apoptosis in PC3 metastatic prostate cancer cell line and shows anti-cancer activity in a xenograft animal model. Brit J Cancer, 86, 1197-203

Li Y, Rizvi SMA, Brown J, Cozzi PJ, Qu CF, Ow KT, Tam PN, Perkins AC, Russell PJ, Allen BJ. (2004a) Antigenic expression of human prostate cancer cell lines and primary prostate cancer sections for in vitro multiple targeted alpha therapy with Bi-213 conjugates. Int J Radiat Oncol Biol Phys: $60 / 3 ; 896-908$

Li, Y., Cozzi, P.J., Qu, C.F., Zhang, D.Y., Abbas Rizvi, S.M., Raja, C. \& Allen BJ (2004b). Cytotoxicity of human prostate cancer cell lines in vitro and induction of apoptosis using 213BiHerceptin alpha-conjugate. Cancer Letters, 205, 161-71.

Miederer, M., Seidl,C,Beyer, G_J, Charlton,D E, Vrasjes-Duric,S, Comor,J J, Huber,R, Nikula,T, Apostolidis,C, Schumacher,C, Becker,K_F, Senekowitsch-Schmidtke,R. (2003). Comparison of the radiotoxicity of two alpha-particle-emitting immunoconjugates, Terbium-149 and Bismuth-213, directed against a tumor-specific, exon 9 deleted (d9) E-cadherin adhesion protein. Radiat Res, 159, $612-20$.

Nielsen A, Scarlett CJ, Samra JS, Gill A, Li Y, Allen BJ, Smith RC.(2005) Significant overexpression of urokinase-type plasminogen activator (UPA) in pancreatic adenocarcinoma using realtime quantitative RT-PCR. J Gastroenterology Hepathology. 20(2):256-63.

Nilsson, S., Larsen,R H, Fosså,S D, Balteskard,L, Borch,K W, Westlin,J E, Salberg,G, Bruland O S (2005). First Clinical Experience with alpha-Emitting Radium-223 in the Treatment of Skeletal Metastases. Clin Cancer Res, 11, 4451-59. 
Nilsson S, Tranzen L, Parker C et al (2007). Bone-targeted radium-233 in symptomatic, hormone refractory prostatwe cancer: a randomised, multicentre, placebo-controlled phase II study. Lancet Oncol 8; 587-594.

Qu Chang Fa, Li Y, Song E, Rizvi SMA, Zhang D, Samra J, Smith R, Perkins AC, Apostolidis C, Allen BJ. (2004) MUC1 expression in primary and metastatic pancreatic cancer cells for in vitro treatment by ${ }^{213} \mathrm{Bi}-\mathrm{C} 595$ radioimmunoconjugate. Brit J Cancer 91; 2086-2093

Qu CF, Song EY, Li Y, Rizvi SMA, Raja C, Smith R, Morgenstern A, Apostolidis C, Allen BJ. (2005a) Preclinical study of ${ }^{213} \mathrm{Bi}$ labeled PAI2 for the control of micrometastatic pancreatic cancer. Clin Exp Metastasis 22; 575-586

Qu, C.F., Song, E.Y., Li, Y., Rizvi, S.M., Raja, C., Smith, R., Morgenstern, A., Apostolidis, C. \& Allen BJ (2005b). Pre-clinical Study of ${ }^{213}$ Bi Labeled PAI2 for the Control of Micrometastatic Pancreatic Cancer. Clin Exp Metastasis 22, 575-86.

Raja C, Graham P, Rizvi SMA, Song E, Goldsmith H, Thompson J, Bosserhoff A, Morgenstern A, Apostolidis C, Kearsley JH, Reisfeld R, Allen BJ. (2007) Interim analysis of toxicity and response in Phase 1 trial of systemic targeted alpha therapy for metastatic melanoma. Cancer Biol Therapy 6:6, 846-52

Rizvi SMA, Sarkar S, Goozee G, Allen BJ. (2000) Radioimmunoconjugates for targeted alpha therapy of malignant melanoma. Melanoma Res 10; 281-289

Rizvi, S.M.A., Allen,B J, Tian Z, Li,Y, Goozee,G, Sarkar,S. (2001). In vitro and preclinical studies of targeted alpha therapy for colorectal cancer. Colorectal Disease, 3, 345-353.

Rizvi, S.M.A., Henniker, A.J., Goozee, G. \& Allen BJ (2002). In vitro testing of the leukaemia monoclonal antibody WM-53 labeled with alpha and beta emitting radioisotopes. Leukemia Res 26, $37-43$.

Rizvi, S.M.A., Sarkar, S., Goozee, G. \& Allen BJ (2000). Radioimmunoconjugates for targeted alpha therapy of malignant melanoma. Melanoma Research, 10, 281-9.

Schmidt, D., Neumann,F, Antke,C, et al. (2004). Phase 1 Clinical Study on Alpha-Therapy for NonHodgkin Lymphoma. In 4th Alpha-Immunotherapy Symposium Morgenstern A, Ed. ITU: Dusseldorf, Germany; pp 12

Song YJ, Qu CF, Rizvi SMA, Li Y, Robertson G, Raja C, Morgenstern A, Apostolidis C, Perkins AC, Allen BJ. (2006) Cytotoxicity of PAI2, C595 and Herceptin Vectors labeled with the Alpha-Emitting Radioisotope Bismuth-213 for Ovarian Cancer Cell Monolayers and Clusters. Cancer Letters 234, 2,176-183.

Song EY, Rizvi SMA, Qu CF, Raja C, Morgenstern A, Apostolidis C, Allen BJ. (2007)

Pharmacokinetics and toxicity of ${ }^{213} \mathrm{Bi}$-labelled PAI2 in preclinical targeted alpha therapy for cancer. Cancer Biol Therapy 6 (6) 898-904

Song EY, Rizvi SMA, Raja C, Qu Changfa, Yuen J, Morgenstern A, Apostolidis C, Allen BJ. (2008a) The cytokinesis-block assay as a biological dosimeter for targeted alpha therapy. Phys Med Biol. 53, 319-328

Song EY, Qu CF, Rizvi SMA, Raja C, Beretov J, Morgenstern A, Apostolidis C, Perkins A, Allen BJ. (2008b) Bismuth-213 radioimmunotherapy with C595 anti-MUC1 monoclonal antibody in an ovarian cancer ascites model. Cancer Biol Therapy 7, 76-80 
TAT 2011: 7th Symposium on targeted Alpha Therapy, Berlin July 17-19

Xue A, Scarlett CJ, Jackson CJ, Allen BJ, Smith RC.(2008) Prognostic significance of growth factors and the urokinase-type plasminogen activator system in pancreatic ductal adenocarcinoma. Pancreas 36(2):160-7.

Zalutsky, MR, Bigner, DD. (1996). Radioimmunotherapy with alpha-emitting radioimmunoconjugates. Acta Oncologia 35, 373-9

Zalutsky, M.R. (2005). Current status of therapy of solid tumours: brain tumour therapy. . J Nucl Med, 46, 151S-156S. 